\title{
Delta and Omega electromagnetic form factors in a covariant three-body approach
}

\author{
Hèlios Sanchis Alepuz* \\ Justus-Liebig-Universität Giessen \\ E-mail: helios.sanchis-alepuzatheo.physik.uni-giessen.de
}

\section{Reinhard Alkofer}

Institut für Physik, Karl-Franzens-Universität, Universitätsplatz, 5, A-8010 Graz, Austria

E-mail: reinhard.alkofereuni-graz.at

\section{Richard Williams}

Institut für Physik, Karl-Franzens-Universität, Universitätsplatz, 5, A-8010 Graz, Austria

E-mail: richard.williamseuni-graz.at

\begin{abstract}
We study baryons as three-body systems using the QCD degrees of freedom in the framework of covariant Bethe-Salpeter equations. The interaction among quarks is reduced to a vector-vector interaction via a single dressed-gluon exchange (Rainbow-Ladder truncation). The formalism allows for the study of the hadron spectrum as well as their internal properties. We will present the calculation of electromagnetic properties of spin-3/2 baryons. The model independent features of our results are assessed using two different models for the dressings.
\end{abstract}

Xth Quark Confinement and the Hadron Spectrum

8 - 12 October 2012

TUM Campus Garching, Munich, Germany

\footnotetext{
* Speaker.
} 


\section{Introduction}

Whereas the internal structure of the nucleon is rather well established experimentally, this is not the case for the next baryonic state, the $\Delta(1232)$. The reason is its unstable nature which makes the study of its properties a challenging task. Insight into the $\Delta$ 's internal structure can be gained by studying its electromagnetic properties.

Since the $\Delta$ is a spin-3/2 particle, its electromagnetic structure is characterized by four electromagnetic form factors; these are the electric monopole $G_{E 0}$, electric quadrupole $G_{E 2}$, magnetic dipole $G_{M 1}$ and magnetic octupole $G_{M 3}$. They are functions of the photon momentum $Q$, their respective values at $Q=0$ are the static electromagnetic moments of the baryon. Experimentally, only the magnetic dipole moments (and, of course, the electric charge) are known for the $\Delta^{+}$and $\Delta^{++}$, albeit with large errors. Indirectly, the study of the $\Delta \rightarrow N \gamma$ decay indicates a deviation of the $\Delta$ from sphericity and therefore a non-vanishing value for $G_{E 2}$.

We present here a calculation of the electromagnetic moments using a covariant Bethe-Salpeter approach. This approach provides a unified quantum-field-theoretical description of meson and baryon properties (see e.g., Ref. [1] and references therein). With the flavor-blind interaction kernels we currently use, the $\Omega(1672)$ is identical to the $\Delta$ but calculated at higher quark mass. Therefore we present also its electromagnetic moments. A more complete study of the $\Delta$ and $\Omega$ electromagnetic properties at non-vanishing momentum transfer will be presented elsewhere [2].

\section{Framework}

In this section we briefly describe the calculation of baryon properties using a covariant threebody Bethe-Salpeter approach; for details we refer to Refs. [2, 3, 4, 5] and references therein.

All the information about the baryon structure is encoded in the Faddeev amplitudes $\Psi$. They are obtained by solving the covariant Faddeev equation

$$
\begin{aligned}
\Psi_{\alpha \beta \gamma \mathscr{I}}(p, q, P)=\int_{k} & {\left[\widetilde{K}_{\beta \beta^{\prime} \gamma \gamma^{\prime}}(k) S_{\beta^{\prime} \beta^{\prime \prime}}\left(k_{2}\right) S_{\gamma^{\prime} \gamma^{\prime \prime}}\left(\tilde{k}_{3}\right) \Psi_{\alpha \beta^{\prime \prime} \gamma^{\prime \prime} \mathscr{I}}\left(p^{(1)}, q^{(1)}, P\right)\right.} \\
& +\widetilde{K}_{\alpha \alpha^{\prime} \gamma \gamma^{\prime}}(-k) S_{\gamma^{\prime} \gamma^{\prime \prime}}\left(k_{3}\right) S_{\alpha^{\prime} \alpha^{\prime \prime}}\left(\tilde{k}_{1}\right) \Psi_{\alpha^{\prime \prime} \beta \gamma^{\prime \prime}} \mathscr{I}\left(p^{(2)}, q^{(2)}, P\right) \\
& \left.+\widetilde{K}_{\alpha \alpha^{\prime} \beta \beta^{\prime}}(k) S_{\alpha^{\prime} \alpha^{\prime \prime}}\left(k_{1}\right) S_{\beta^{\prime} \beta^{\prime \prime}}\left(\tilde{k}_{2}\right) \Psi_{\alpha^{\prime \prime} \beta^{\prime \prime} \gamma \mathscr{I}}\left(p^{(3)}, q^{(3)}, P\right)\right],
\end{aligned}
$$

with $p$ and $q$ being the relative momenta, as depicted in Fig. 1 and defined in [2], and $P$ is the total momentum. $S_{\alpha^{\prime} \alpha^{\prime \prime}}\left(k_{1}\right)$ is the fully dressed quark propagator. Here we use a Ladder truncation for the two-body interaction kernel

$$
\widetilde{K}=4 \pi Z_{2}^{2} \frac{\alpha_{\mathrm{eff}}\left(k^{2}\right)}{k^{2}} T_{\mu v}(k) \gamma^{\mu} \otimes \gamma^{v}
$$

with $Z_{2}$ being the quark renormalization constant, $T_{\mu v}(k)$ the $k$-transverse projector and $k$ the gluon momentum. In addition we neglect three-body irreducible interactions.

Eq. (2.1) requires the fully dressed quark propagator and the effective interaction $\alpha_{\text {eff }}$ in the two-body interaction kernel as input. For consistency, the quark propagators are obtained by solving the quark Dyson-Schwinger equation (DSE) in the Rainbow truncation such that also for these equations $\alpha_{\text {eff }}$ is the only needed input. 


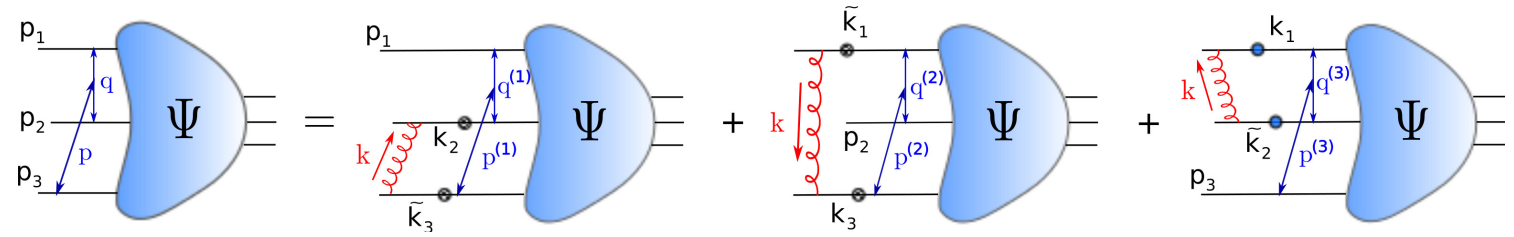

Figure 1: Covariant Faddeev equation in the Rainbow-Ladder truncation.

The Rainbow-Ladder truncation thus relies on the choice of an effective interaction $\alpha_{\text {eff }}$. To learn about the model dependence of our results we compare two different ansätze: the first one is the Maris-Tandy model $[6,7]$ which was originally invented to optimally reproduce light pseudoscalar observables; the second is a model designed to obtain the $U_{A}(1)$ anomaly enhanced $\eta^{\prime}$ mass via the Kogut-Susskind mechanism [8,9] and also to describe correctly pseudoscalar meson properties [10]. Both models, which we denote as models I and II, are compared in Figure 2; for a detailed description of both of them we refer to Refs. [2, 5].

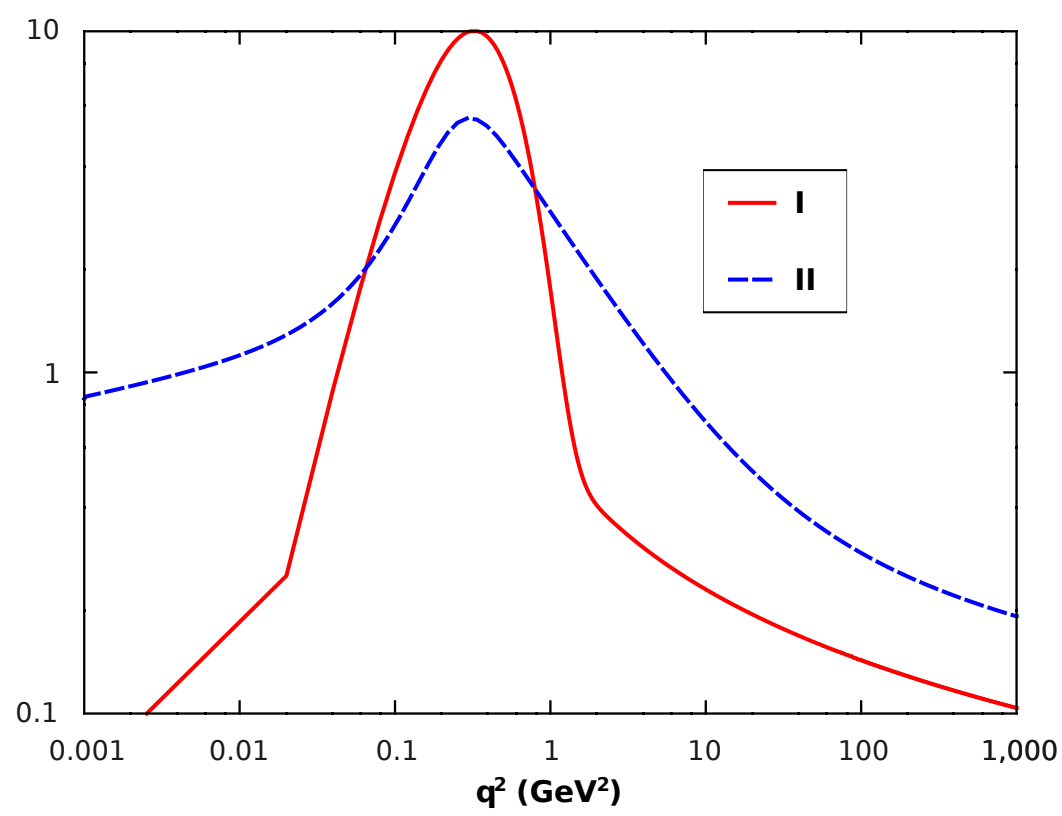

Figure 2: Comparison of the behavior of $\alpha_{\mathrm{eff}}\left(q^{2}\right)$ for model I [6, 7] and model II [10] used for the effective interaction.

Note that Eq. (2.1) determines besides the Faddeev amplitudes also the baryon mass: Only for the bound state mass $P^{2}=-M_{B}^{2}$ it will be fulfilled. In addition we want to remark that being constructed from the tensor product of three Dirac spinors the number of independent tensor components of the Faddeev amplitudes is quite large, 64 for the nucleon and 128 for the $\Delta$ or $\Omega$. Once the Faddeev amplitudes are obtained for given baryon quantum numbers further information about the considered baryon can be extracted. For example, one can use them to calculate the baryonic 
electromagnetic current using the equation

$$
\begin{aligned}
J_{\mathscr{I}^{\prime} \mathscr{I}}^{\mu}=\int_{p} \int_{q} \bar{\Psi}_{\beta^{\prime} \alpha^{\prime} \mathscr{I}^{\prime} \gamma^{\prime}}\left(p_{f}^{\{1\}}, q_{f}^{\{1\}}, P_{f}\right)[ & \left.\left(S\left(p_{1}^{f}\right) \Gamma^{\mu}\left(p_{1}, Q\right) S\left(p_{1}^{i}\right)\right)_{\alpha^{\prime} \alpha} S_{\beta^{\prime} \beta}\left(p_{2}\right) S_{\gamma^{\prime} \gamma}\left(p_{3}\right)\right] \times \\
& \left(\Psi_{\alpha \beta \gamma \mathscr{I}}\left(p_{i}^{\{1\}}, q_{i}^{\{1\}}, P_{i}\right)-\Psi_{\alpha \beta \gamma \mathscr{I}}^{\{1\}}\left(p_{i}^{\{1\}}, q_{i}^{\{1\}}, P_{i}\right)\right) \\
+\int_{p} \int_{q} \bar{\Psi}_{\beta^{\prime} \alpha^{\prime} \mathscr{I}^{\prime} \gamma^{\prime}}\left(p_{f}^{\{2\}}, q_{f}^{\{2\}}, P_{f}\right)[ & \left.S_{\alpha^{\prime} \alpha}\left(p_{1}\right)\left(S\left(p_{2}^{f}\right) \Gamma^{\mu}\left(p_{2}, Q\right) S\left(p_{2}^{i}\right)\right)_{\beta^{\prime} \beta} S_{\gamma^{\prime} \gamma}\left(p_{3}\right)\right] \times \\
& \left(\Psi_{\alpha \beta \gamma \mathscr{I}}\left(p_{i}^{\{2\}}, q_{i}^{\{2\}}, P_{i}\right)-\Psi_{\alpha \beta \gamma \mathscr{I}}^{\{2\}}\left(p_{i}^{\{2\}}, q_{i}^{\{2\}}, P_{i}\right)\right) \\
+\int_{p} \int_{q} \bar{\Psi}_{\beta^{\prime} \alpha^{\prime} \mathscr{I}^{\prime} \gamma^{\prime}}\left(p_{f}^{\{3\}}, q_{f}^{\{3\}}, P_{f}\right) & {\left[S_{\alpha^{\prime} \alpha}\left(p_{1}\right) S_{\beta^{\prime} \beta}\left(p_{2}\right)\left(S\left(p_{3}^{f}\right) \Gamma^{\mu}\left(p_{3}, Q\right) S\left(p_{3}^{i}\right)\right)_{\gamma^{\prime} \gamma}\right] \times } \\
& \left(\Psi_{\alpha \beta \gamma \mathscr{I}}\left(p_{i}^{\{3\}}, q_{i}^{\{3\}}, P_{i}\right)-\Psi_{\alpha \beta \gamma \mathscr{I}}^{\{3\}}\left(p_{i}^{\{3\}}, q_{i}^{\{3\}}, P_{i}\right)\right),
\end{aligned}
$$

which is depicted in Fig. 3. We refer again to Ref. [2] for a definition of the kinematical variables. From the current $J$ one can extract the electromagnetic form factors via the appropriate contractions (see, e.g. [11]). The non-perturbative quark-photon vertex required to calculate the electromagnetic current is obtained by solving the vertex inhomogeneous Bethe-Salpeter equation in the RainbowLadder truncation.

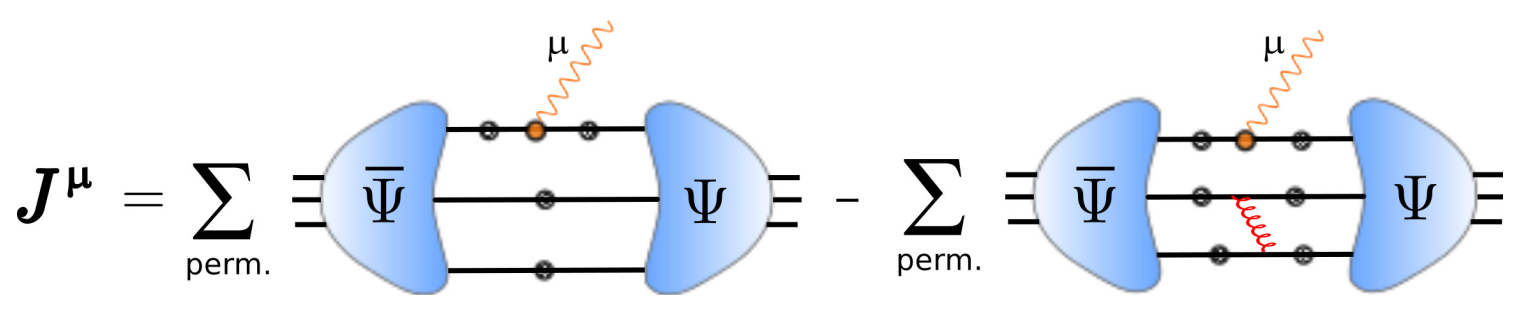

Figure 3: Calculation of the baryon electromagnetic current in the Rainbow-Ladder truncation.

\section{Results}

The electric monopole moment is not very interesting since it gives the electric charge of the baryon and it is fixed by the normalization of the Faddeev amplitudes. Nevertheless, related to its evolution with respect to the photon momentum is the electric charge radius

$$
\left\langle r_{E 0}^{2}\right\rangle=-\left.\frac{6}{G_{E 0}(0)} \frac{d G_{E 0}}{d Q^{2}}\right|_{Q^{2}=0} .
$$

We show our results for the $\Delta$ in Table 1 and for the $\Omega$ in Table 2 and compare to lattice results at different pion masses $[14,15,16]$. One sees that there seems to be a significant disagreement between our results and the corresponding lattice data. However, a large part of this disagreement is simply due to the different values obtained for the decisive scale of the problem, namely, the baryon mass. This disagreement is, therefore, largely reduced by comparing the dimensionless quantity $\left\langle r_{E 0}^{2}\right\rangle M_{\Delta}^{2}$. In this case model I gives a result consistent with lattice data, whereas model II overestimates it somewhat. 


\begin{tabular}{l|cc|ccc|c} 
& F-I & F-II & DW1 & DW2 & DW3 & Exp. \\
\hline \hline$M_{\Delta}(\mathrm{GeV})$ & 1.22 & 1.22 & $1.395(18)$ & $1.559(19)$ & $1.687(15)$ & $1.232(2)$ \\
\hline$\left\langle r_{E 0}^{2}\right\rangle\left(\mathrm{fm}^{2}\right)$ & 0.50 & 0.61 & $0.373(21)$ & $0.353(12)$ & $0.279(6)$ & \\
\hline$\left\langle r_{E 0}^{2}\right\rangle M_{\Delta}^{2}$ & 0.75 & 0.91 & $0.726(36)$ & $0.858(25)$ & $0.794(14)$ & \\
\hline$G_{M 1}(0)$ & 2.38 & 2.77 & $2.35(16)$ & $2.68(13)$ & $2.589(78)$ & $3.54_{-4.72}^{+4.59}$ \\
\hline$G_{E 2}(0)$ & -0.67 & -0.99 & $-0.87(67)$ & $-1.68(88)$ & $-0.71(49)$ & \\
\hline
\end{tabular}

Table 1: Comparison of static results for the $\Delta^{+}$. We compare our results for models I and II (F-I and F-II, respectively) with a lattice calculation with dynamical Wilson fermions at $m_{\pi}=384 \mathrm{MeV}(\mathrm{DW} 1), m_{\pi}=$ $509 \mathrm{MeV}$ (DW2) and $m_{\pi}=691 \mathrm{MeV}$ (DW3) [14, 15]. For $G_{M 1}(0)$ we also compare with the experimental value $[12,13]$.

The magnetic dipole moment $\mu$ is the only electromagnetic property (besides the electric charge) that has been measured experimentally $[12,13]$, both for the $\Delta$ and for the $\Omega$. We show in Tables 1 and 2 the dimensionless quantity $G_{M 1}(0)$ which is related to the magnetic dipole via $\mu=G_{M 1}(0) e / 2 M$. In the case of the $\Delta$, our result is consistent with the experimental value due to the large uncertainties in the latter; however, the experimental value for the $\Omega$ is very accurately measured and our results from the Rainbow-Ladder Faddeev equation clearly disagree. We interpret this disagreement to be mainly caused by the fact that in the chosen truncation effects of meson dressings of the baryon quark core are absent. ${ }^{1}$ The comparison with lattice data is more favorable for the $\Delta$ since, presumably, due to the high pion mass in lattice calculations pion cloud effects are also suppressed in the lattice calculations.

The last quantity we show is $G_{E 2}(0)$, which is related to the magnetic quadrupole moment $\mathscr{Q}$ via $\mathscr{Q}=G_{E 2}(0) e / M^{2}$. In the Breit frame, it can be interpreted as a measure of the deformation from sphericity of the electric charge distribution and is therefore a quantity of significant interest. The sign of the quadrupole moment thus indicates the overall shape of the distribution; our calculations agree with lattice that both the $\Delta$ and $\Omega$ feature an oblate $(\mathscr{Q}<0)$ charge distribution. At the quantitative level, as with the magnetic dipole, we observe that lattice results and ours agree reasonably well for the $\Delta$ (although in this case statistical errors for lattice data are very high) and for the Omega the difference becomes more marked.

The shape of the magnetic dipole distribution is indicated by the magnetic octupole moment $\mathscr{O}$. Although in the complete calculation presented in [2] we present evidence that $\mathscr{O}$ is likely positive and, therefore, the magnetic dipole distribution is prolate, this quantity is numerically very sensitive and we cannot provide a truely reliable value at $Q=0$.

\footnotetext{
${ }^{1}$ In case of the $\Omega$ one expects effects mostly from a kaon cloud.
} 


\begin{tabular}{l|cc|ccc|c|} 
& F-I & F-II & DW1 & DW2 & Hyb. & Exp. \\
\hline \hline$M_{\Omega}(\mathrm{GeV})$ & 1.65 & 1.80 & $1.76(2)$ & $1.77(3)$ & $1.78(3)$ & 1.672 \\
\hline$<r_{E 0}^{2}>\left(\mathrm{fm}^{2}\right)$ & 0.27 & 0.27 & $0.355(14)$ & $0.353(8)$ & $0.338(9)$ & \\
\hline$<r_{E 0}^{2}>M_{\Omega}^{2}$ & 0.74 & 0.89 & $0.726(36)$ & $0.858(25)$ & $0.794(14)$ & \\
\hline$G_{M 1}(0)$ & -2.41 & -2.71 & $-3.443(173)$ & $-3.601(109)$ & $-3.368(80)$ & $-3.52(9)$ \\
\hline$G_{E 2}(0)$ & 0.54 & 0.75 & $0.959(41)$ & & $0.838(19)$ & \\
\hline
\end{tabular}

Table 2: Comparison of static results for the $\Omega^{-}$. We compare our results for models I and II (F-I and F-II, respectively) with a lattice calculation with dynamical Wilson fermions at $m_{\pi}=297 \mathrm{MeV}$ (DW1), $m_{\pi}=330 \mathrm{MeV}$ (DW2) and with a hybrid action at $m_{\pi}=353 \mathrm{MeV}(\mathrm{Hyb})$ [16]. For $G_{M 1}(0)$ we also compare to the experimental value [13].

\section{Summary}

We have presented results for the electromagnetic moments of the $\Delta$ and $\Omega$ baryons calculated from a self-consistent system of Dyson-Schwinger equations for the quark propagators and the quark-photon vertex as well as three-body Bethe-Salpeter type equations for the baryon quark core. We truncated the system to a single dressed-gluon exchange and used two different models for the effective interaction to obtain some rough estimate of the model dependence of the obtained results. These are in reasonable agreement with corresponding lattice data. However, in the case of the magnetic moment of the $\Omega$, the only case considered here where sufficiently precise experimental data is available, the obtained results display a qualitatively correct behaviour but are on the quantitative level unsatisfactory.

Such disagreements might be related to the employed Faddeev approximation (i.e., neglecting three-particle irreducible interactions), deficiencies in the Rainbow-Ladder approximation and the modeled interaction and/or neglecting mesonic dressing effects. To this end we note that for mesonic bound states significantly more sophisticated truncation schemes have been successfully employed, see e.g., Refs. [17, 18, 19, 20], and can be, in principle, also applied to baryons as three-quark bound states.

\section{Acknowledgements}

We are grateful to the organizers of the Xth Quark Confinement and the Hadron Spectrum conference for all their efforts which made this conference possible.

We thank Gernot Eichmann, Christian S. Fischer and Selym Villalba-Chavez for helpful discussions.

During the course of this work HSA was funded by the Austrian Science Fund, FWF, under project P20592-N16 and supported by the Doctoral Program on Hadrons in Vacuum, Nuclei, and Stars (FWF DK W1203-N16); RW acknowledges funding by FWF under project M1333-N16. Further 
support by the European Union (HadronPhysics2 project "Study of strongly-interacting matter") is acknowledged.

\section{References}

[1] G. Eichmann, PhD Thesis, U. Graz 2009 [arXiv:0909.0703 [hep-ph]].

[2] H. Sanchis-Alepuz, R. Williams and R. Alkofer, in preparation.

[3] G. Eichmann et al., Phys. Rev. Lett. 104 (2010) 201601 [arXiv:0912.2246 [hep-ph]].

[4] G. Eichmann, Phys. Rev. D 84 (2011) 014014 [arXiv:1104.4505 [hep-ph]].

[5] H. Sanchis-Alepuz et al., Phys. Rev. D 84 (2011) 096003 [arXiv:1109.0199 [hep-ph]]; PoS QNP 2012 (2012) 112 [arXiv:1206.6599 [hep-ph]]; PoS QCD-TNT-II (2011) 041 [arXiv:1112.3214 [hep-ph]].

[6] P. Maris and C. D. Roberts, Phys. Rev. C 56 (1997) 3369.

[7] P. Maris and P. C. Tandy, Phys. Rev. C 60 (1999) 055214.

[8] J. B. Kogut and L. Susskind, Phys. Rev. D 10 (1974) 3468.

[9] L. von Smekal, A. Mecke and R. Alkofer, arXiv:hep-ph/9707210.

[10] R. Alkofer, C. S. Fischer and R. Williams, Eur. Phys. J. A 38 (2008) 53.

[11] D. Nicmorus, G. Eichmann and R. Alkofer, Phys. Rev. D 82 (2010) 114017 [arXiv:1008.3184 [hep-ph]].

[12] M. Kotulla et al., Phys. Rev. Lett. 89 (2002) 272001 [nucl-ex/0210040].

[13] J. Beringer et al. [Particle Data Group Collaboration], Phys. Rev. D 86 (2012) 010001.

[14] C. Alexandrou et al., Nucl. Phys. A 825 (2009) 115 [arXiv:0901.3457 [hep-lat]].

[15] C. Alexandrou et al., PoS CD 09 (2009) 092 [arXiv:0910.3315 [hep-lat]].

[16] C. Alexandrou et al., Phys. Rev. D 82 (2010) 034504 [arXiv:1006.0558 [hep-lat]].

[17] C. S. Fischer, D. Nickel and J. Wambach, Phys. Rev. D 76 (2007) 094009 [arXiv:0705.4407 [hep-ph]].

[18] C. S. Fischer and R. Williams, Phys. Rev. D 78 (2008) 074006 [arXiv:0808.3372 [hep-ph]].

[19] L. Chang and C. D. Roberts, Phys. Rev. Lett. 103 (2009) 081601 [arXiv:0903.5461 [nucl-th]].

[20] C. S. Fischer and R. Williams, Phys. Rev. Lett. 103 (2009) 122001 [arXiv:0905.2291 [hep-ph]]. 